\title{
An Online System for Detecting Bending in a Pallet Car
}

\author{
Ahmad Pouramini \\ Computer Engineering Department \\ Sirjan University of Technology, Iran
}

\begin{abstract}
Image processing techniques are widely used to detect defects in industrial equipment. In this paper, an online system is presented to detect bending in pallet cars of a travelling grate conveyor used in sintering machines. If bending in several pallet cars exceeds a specified limit, it can stop the production line. Therefore, an early and precise diagnosis is required. The system consists of a camera in a specific position to monitor the pallet cars and provide an online video. A method is presented to detect and extract an appropriate image of a pallet car from this video. The image is then processed to detect bending of the pallet car's middle frame and measure the degree of bending. Particularly, edge detection methods and Hough transform are used to locate and measure the curvature. The experimental results show a precision of $98 \%$ and a recall of $100 \%$ for the detection method.
\end{abstract}

\section{General Terms}

Image Processing

\section{Keywords}

Defect Detection, Hough Transform

\section{INTRODUCTION}

Image processing is one of the mostly increasing areas in computer science. Digital image processing is used to extract various features from images [11]. This is done by computers automatically without or with little human intervention. One of the most important operations on digital image is to automatically identify and classify various kinds of defects in industrial equipment and products [10. 3]. In this paper an application of image processing is presented for detecting defects in a pallet car of a sintering machine used in pelletizing industry.

In pelletizing process, after preparing green pellets, they are transported on a special conveyor, called traveling grate, through a sintering machine where they are dried, burned and cooled. Traveling grate consists of a chain of pallet cars rolling on rails. The sinter is discharged at the tipping station, where the pallet cars are lowered from the upper strand to the return track by gear rims and curved rails. Then, they return to the charging station to be charged again with green pellets.

Figure 1 shows an isolated pallet car. It consists of four main parts namely two side walls, one steel cast middle frame and four traveling rollers. Bending can occur on the middle frame

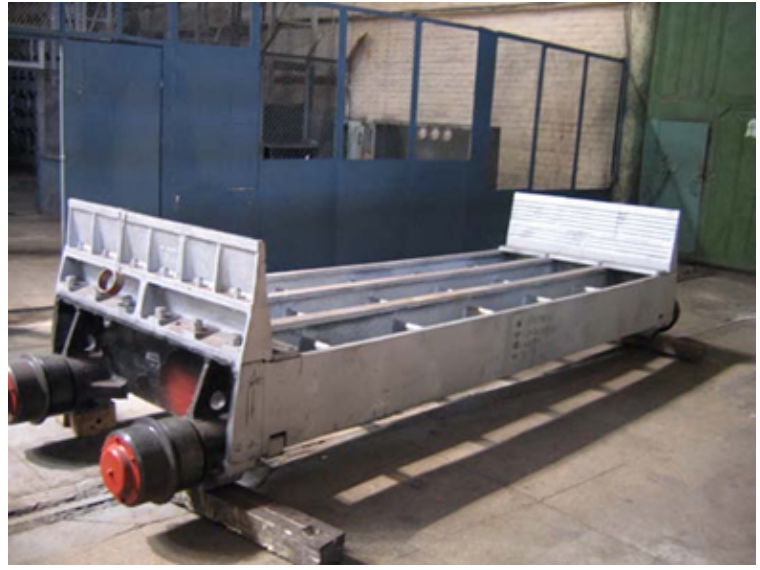

Fig. 1. A typical isolated pallet car.

during operation due to the high temperature of the furnace and axial strain. Excessive bending can cause the pallet car to become engaged with the underlying structure, increasing the force required to rotate the conveyor. At the extreme, it can stop the conveyor's movement. The bending may be slight or severe; therefore, a precise method is required to detect a smooth curvature in the body of a pallet car. The first stage is to acquire a suitable image from the middle frame of each pallet car. In the next section, a method to accomplish this is proposed.

\section{IMAGE ACQUISITION}

The system consists of a camera for acquisition of an online video from the in-operation conveyor. The only place for capturing an image from the middle side of each pallet car is before the charging station, where the pallet cars are rotating on the curved rails. The rotation opens up a gap between two successive pallet cars where the middle side of the first pallet car is observable. Figure 2 shows two pictures taken from this view. The best time to acquire a suitable frame is when the pallet car is in horizontal position (left picture of Figure 2). This problem is a case of event detection and visual object tracking whose core is to robustly estimate the motion state (i.e., location, orientation, size, etc.) of a target object in each frame of an input image sequence [5, 7].

To track the pallet car movement and capture the desired frame, the color changes of three squared regions in consecutive frames are tracked (see Figure 2). First, the color image is converted to 

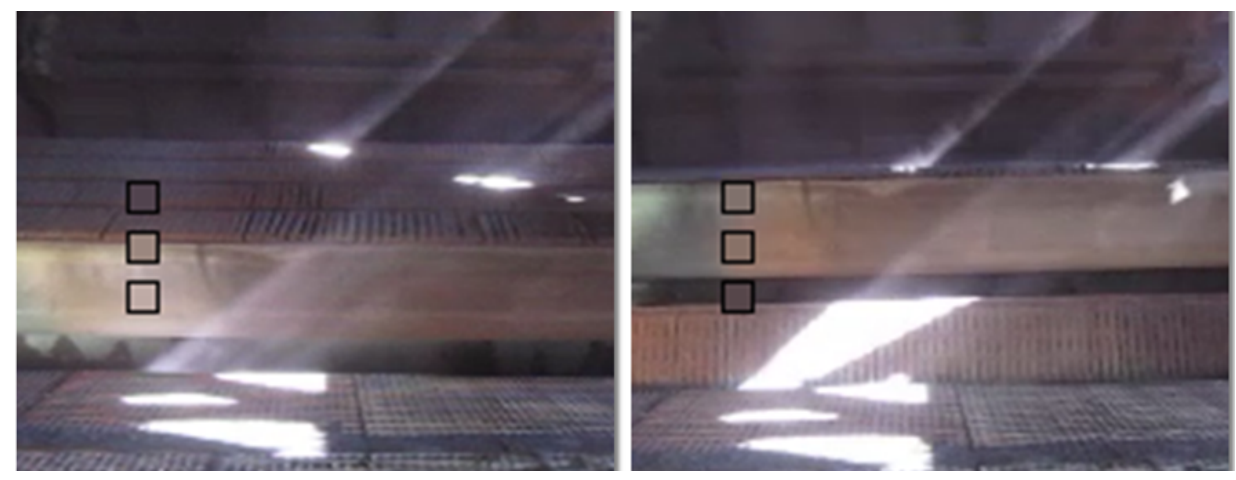

Fig. 2. The front view of pallet cars before charging station

a grayscale image. Then, for each square, its average gray value is calculated. As shown in Figure 2, when the pallet car is fully horizontal, the color of the first and second squares is very close to each other because they both belongs to the same surface. At the same time, the third square is much darker than the other squares because it falls into the gap area before the pallet car. We employ two thresholds to detect this arrangement. Suppose that the average gray value of the $n$th square is specified by MeanGray $y_{n}$. Then a video frame satisfying the following conditions is extracted:

$$
\begin{gathered}
\text { TargetFrame }=\left(\text { MeanGray }_{2}-\text { MeanGray }_{1}<T_{1}\right) \text { and } \\
(\text { MeanGray } \\
- \text { MeanGray }
\end{gathered}
$$

The first condition states that the difference between the average gray value of the first and second squares must be less than a specified threshold. It means that their color is close enough to each other to be considered as one surface. The second condition states that the average gray value difference of the third and second squares must be greater than a specified threshold. It means that the third square is sufficiently darker than the second square. One may use the average value of the first and second squares instead of only the second square. When these conditions are met, the associated video frame is extracted and is passed to the next stage.

The advantage of this method is that it is independent of any specific color of the middle frame of the pallet cars. The color of the middle frame can vary depending on the light environment and the pallet cars' surface conditions, making the detection solely based on a specific color difficult.

\section{BENDING MEASUREMENT}

The next stage is to detect and measure the bending of a pallet car in the image resulting from the previous stage. Figure 3 shows an image extracted from the video. As shown, the pallet car is in horizontal position and its middle frame is completely visible. The pallet car shown in Figure 3 is slightly bent. This bending must be detected and its degree must be determined. For this purpose, first, a series of image processing transformations is applied to the image.

Since there is no useful color information in the image to distinguish visual features, it is first converted to a grayscale image. Then, an edge detection method is employed to distinguish the edges of the pallet car. There mainly exist several edge detection methods (Sobel, Prewitt, Roberts, Canny) [8 [14, 15]. These methods have been proposed for detecting transitions in images. For this work, the Canny edge detection algorithm, which is known as the optimal edge detector, was selected [1 9]. In order to discard small edges that may be due to noise and keep the strongest ones, a threshold of 0.25 was selected to be applied to the Canny method in Matlab (The range is from 0 (all edges) to 0.99 ... (only the strongest ones) [9]. The result of applying the Canny method to the image of Figure 3 is shown in Figure 4. As can be observed, the edges of the pallet car, which are represented by white pixels, are partially detected.

The next stage is to detect bending and to measure the degree of bending. For this purpose, a region in the image that includes the top edge is identified. Then, a curve is fitted through the edge pixels in this region. The coordinates of such a region cannot be fixed, because minor changes in the angle of the camera, or differences in the positions of different pallet cars require readjustment. Therefore, we detect the approximate location of the top edge in the image. For this purpose, we use the Hough transform. The Hough transform is widely used to detect straight lines in an image [6, 4] 2]. In our application, we first identify all the straight lines in the image. Then, the longest horizontal line in an approximate region around the top edge is selected as the top edge position. A rectangular area with equal distance from this line is selected as the region of interest. This region is later processed to detect the bending of the top edge.

In the following, we briefly describe how the Hough transform works and why it is suitable for our purpose. Hough transform is based on the fact that a line in the x-y Cartesian coordinate system can be mapped onto a point in the rho-theta space.

$$
y=m x+b \text { is mapped onto } r=x \cos \theta+y \sin \theta
$$

where $r$ is the distance from the origin to the closest point on the straight line, and ? (theta) is the angle between the $\mathrm{x}$ axis and the line connecting the origin with that closest point. Now when we a point on the image, and we are not sure whether or not it belongs to a straight line in the image, we just go ahead and plot a point for all possible lines that can pass through that point. That would result in a sinusoidal curve in the rho-theta space. If the point actually does belong to a line, the actual rho-theta coordinate in the rho-theta plane will be reinforced by all points that belong to the line. If we plot an intensity curve of the reinforcement strength (number of curves that cross a point in the rho-theta space), we can see peaks at the values that correspond to possible lines. These points can be isolated and picked up by applying a threshold value.

As can be observed from Figure 3, white pixels that reside on a straight line along the pallet car's top edge are sufficient to 


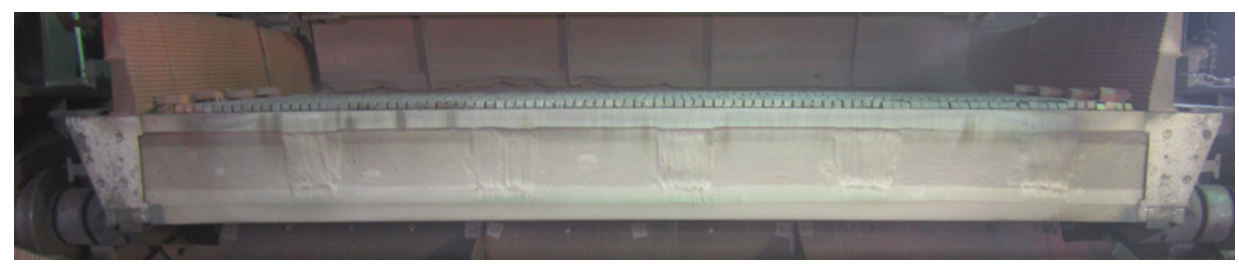

Fig. 3. An image from the front side of the middle frame extracted from the video stream.

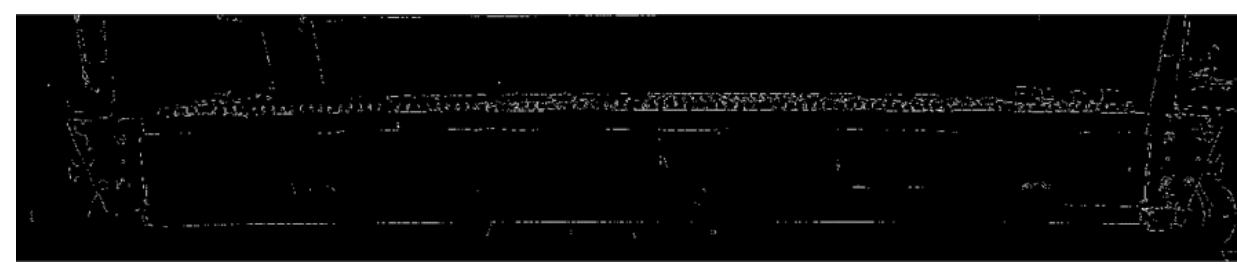

Fig. 4. Applying Canny edge detection method on Figure 3

determine a straight line using Hough transform. In other words, there is a straight line that fits these points. From the list of lines that are provided by Hough transform, the longest horizontal line that resides in an approximate region around the top edge is selected. For example, the row number of such a line must be less than 1000 (the upper area of the image). This technique resulted the line shown in Figure 5 for the image of Figure 4. After identifying the location of this line, a rectangular region along this line is selected as the region of interest (ROI). This region is processed to identify and select a series of white pixels for curve fitting. If $y$ is the row number of this line, a rectangle with the following coordinates is selected:

$$
R O I=R E C T(0, y-100, \text { ImgWidth, } y+100) .
$$

After specifying the region of interest (ROI), an edge detection mask is applied again to the corresponding region in the original grayscale image to detect horizontal edges. In order to detect only very distinct and large horizontal edges, the filter smooths a 50 pixels wide region using a 15-by-50 Prewitt mask [13]. The 7 upper rows of the mask are filled with 1 , and the 7 lower rows are filled with -1 . The center row is filled with 0 .

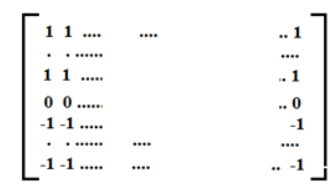

The resulting mask is applied to ROI to obtain an image that include the target horizontal edge. Then, a threshold of 60 is used to identify the strongest points that reside on the detected edge. From these points, the points that belong to a rectangular region around the center of the top edge, with a height of 100 pixels and a width equal to $3 / 4$ of the image width, are selected. The region was restricted so that the curve fitting process dose not mislead by noisy points. Now, a second order polynomial curve is fitted to the selected points. Finding a curve that passes through a set of given points is the problem of interpolation, while finding a curve that passes near a set of given points is the problem of approximation [12]. In this work, by curve fitting we mean an approximation which is the best polynomial fit of degree two (in a least-squares sense) for the selected points. The curve resulting from this process is drawn in Figure 6. The first coefficient of the curve (the coefficient of $x^{2}$ ) which is a monotonic variable, is used to quantify the degree of bending. In Figure 6 this value is 2.55, which is not a negligible value, making the curve distinct from a straight line. As can be observed, this curve adequately fits the top boundary of the pallet car.

\section{EXPERIMENTAL RESULTS}

The proposed method was tested on 100 pallet cars of an in-operation sintering machine to detect the pallet cars with excessive bending. The first coefficient of the resulting curve were compared against a threshold of 5 to detect the pallet cars with excessive bending. The detected pallet cars were manually inspected to measure the system performance. This resulted a precision value of $98 \%$ and a recall value of $100 \%$.

\section{CONCLUSION}

In this paper, an on-line system for automatic detection of bending in the pallet cars of a traveling grate conveyor was presented. After capturing an online video from the front view of the pallet cars, the video is processed to extract a frame displaying the pallet car in horizontal position. Then this image was processed to measure the degree of bending in the middle frame. For this purpose, first, the edges of the frame were detected using Canny method. Then, the Hough transform was used to determine the location of the frame's top edge in the image. We focused the processing on a region around this edge as the region of interest. First, a 15-by-50 Prewitt mask was used to detect large and distinct horizontal edges in the region. Then, the strong horizontal edge pixels around the center of the region were selected and a second order polynomial was fit to them. The resulting curve adequately fits the top boundary of a pallet car and its coefficients can represent the degree of bending. This value was compared against a threshold to detect the pallet cars with excessive bending. Testing the method on 100 pallet cars resulted a precision of $98 \%$ and a recall of $100 \%$. 


\section{REFERENCES}

[1] John Canny. A computational approach to edge detection. IEEE Transactions on pattern analysis and machine intelligence, (6):679-698, 1986.

[2] Opas Chutatape and Linfeng Guo. A modified hough transform for line detection and its performance. Pattern Recognition, 32(2):181-192, 1999.

[3] Christian Demant, C Demant, and Bernd Streicher-Abel. Industrial image processing. Springer, 1999.

[4] Leandro AF Fernandes and Manuel M Oliveira. Real-time line detection through an improved hough transform voting scheme. Pattern Recognition, 41(1):299-314, 2008.

[5] Niels Haering and Niels da Vitoria Lobo. Visual Event Detection, volume 2. Springer Science \& Business Media, 2013.

[6] John Illingworth and Josef Kittler. A survey of the hough transform. Computer vision, graphics, and image processing, 44(1):87-116, 1988.

[7] Xi Li, Weiming $\mathrm{Hu}$, Chunhua Shen, Zhongfei Zhang, Anthony Dick, and Anton Van Den Hengel. A survey of appearance models in visual object tracking. ACM transactions on Intelligent Systems and Technology (TIST), 4(4):58, 2013.

[8] Roman Louban. Image processing of edge and surface defects. Springer, 2009.

[9] Raman Maini and Himanshu Aggarwal. Study and comparison of various image edge detection techniques. International journal of image processing (IJIP), 3(1):1-11, 2009.

[10] Elias N Malamas, Euripides GM Petrakis, Michalis Zervakis, Laurent Petit, and Jean-Didier Legat. A survey on industrial vision systems, applications and tools. Image and vision computing, 21(2):171-188, 2003.

[11] Jim R Parker. Algorithms for image processing and computer vision. John Wiley \& Sons, 2010.

[12] Theodosios Pavlidis. Algorithms for graphics and image processing. Springer Science \& Business Media, 2012.

[13] Judith MS Prewitt. Object enhancement and extraction. Picture processing and Psychopictorics, 10(1):15-19, 1970.

[14] N Senthilkumaran and R Rajesh. Edge detection techniques for image segmentation-a survey of soft computing approaches. International journal of recent trends in engineering, 1(2), 2009.

[15] GT Shrivakshan, C Chandrasekar, et al. A comparison of various edge detection techniques used in image processing. IJCSI International Journal of Computer Science Issues, 9(5):272-276, 2012.

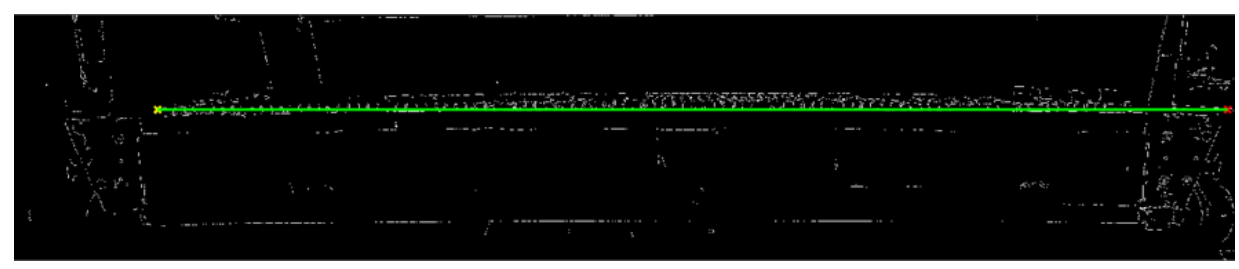

Fig. 5. The line located on the top edge detected by Hough transform. 


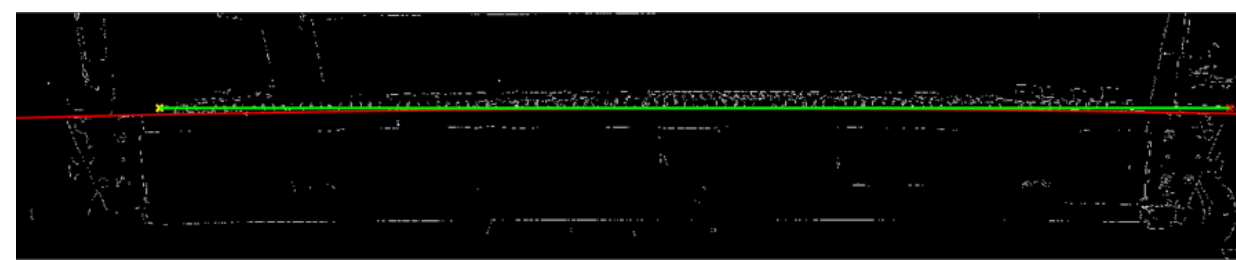

Fig. 6. The curve fitting the top boundary of the pallet car. 